\title{
Obinutuzumab in chronic lymphocytic leukemia: design, development and place in therapy
}

\author{
This article was published in the following Dove Press journal: \\ Drug Design, Development and Therapy \\ 25 January 2017 \\ Number of times this article has been viewed
}

\author{
Othman Al-Sawaf \\ Kirsten Fischer \\ Anja Engelke \\ Natali Pflug \\ Michael Hallek \\ Valentin Goede \\ German CLL Study Group, \\ Department I of Internal Medicine, \\ University Hospital of Cologne, \\ Cologne, Germany
}

Correspondence: Valentin Goede German CLL Study Group, Department I of Internal Medicine, University Hospital of Cologne, Kerpener Straße 62, Cologne, Germany

Email valentin.goede@uk-koeln.de

\begin{abstract}
For decades, treatment of chronic lymphocytic leukemia (CLL) has been based on chemotherapy. This changed when the first CD20 antibody rituximab was introduced. Since 2008, the combination of chemotherapy and CD20 antibodies has become the standard of care for most patients, and a significant fraction of patients had very long-lasting remissions after chemoimmunotherapy. Despite the improvement of response rates and overall survival (OS) by the use of chemoimmunotherapy, most CLL patients will relapse eventually. One approach to achieve more durable responses was the development of obinutuzumab (GA101), a new type of CD20 antibody that has unique molecular and functional characteristics. Obinutuzumab is a type II fully humanized CD20 antibody that binds to a partly different epitope of the CD20 protein than rituximab and due to its glycoengineered design induces greater antibody-dependent cell-mediated cytotoxicity (ADCC). Initial preclinical observations of a more effective B-cell depletion have been successfully reproduced in clinical trials with CLL patients. This review summarizes results of preclinical as well as clinical studies with obinutuzumab and provides an outlook on its future role in the therapy of CLL.
\end{abstract}

Keywords: chronic lymphocytic leukemia, GA101, obinutuzumab, CD20 antibody

\section{Introduction}

Therapy of chronic lymphocytic leukemia (CLL) has been strongly improved by the combined use of chemotherapy and monoclonal antibodies targeting the CD20 antigen. In 2010, the CLL8 trial by the German CLL Study Group (GCLLSG) showed that addition of the type I chimeric IgG1 CD20 antibody rituximab increased progressionfree survival (PFS) and overall survival (OS) when combined to the chemotherapy with fludarabine and cyclophosphamide (FCR). ${ }^{1,2}$ Another well-validated chemoimmunotherapy is bendamustine plus rituximab (BR), which has been shown to be less effective than FCR, but more tolerable with regard to toxicity (particularly in patients older than 65 years). ${ }^{3}$ Single-agent activity of rituximab is only modest in most cases of indolent non-Hodgkin lymphoma; however, it has significant activity in CLL patients at higher dose levels. ${ }^{4}$ Hence, chemoimmunotherapy with rituximab has become the standard of care for most patients with CLL in the upfront setting.

Despite long-lasting remissions after chemoimmunotherapy with a median PFS of $>6$ years in some subgroups, ${ }^{5}$ most patients will eventually relapse after chemoimmunotherapy and may develop chemotherapy- or rituximab-refractory disease. One recent attempt to achieve deeper and long-lasting remissions was to develop novel CD20 antibodies with improved therapeutic efficacy compared to rituximab. Obinutuzumab (formerly GA101) is the first of a new generation of type II glycoengineered CD20 monoclonal antibodies that has been approved for the 
treatment of CLL. Previous review articles have discussed and summarized key progress of developing this antibody. ${ }^{6-8}$ Meanwhile, new as well as updated data for obinutuzumab have emerged with regard to the treatment not only of CLL but also of other B-cell lymphomas. In this study, we recapitulate the initial steps of the development and the particular pharmacological characteristics of obinutuzumab, review currently available data on its use in the clinical setting with focus on recently released results of older and novel trials and provide an outlook on the antibody's future application in the treatment of CLL.

\section{Design and characteristics} Function of CD20 monoclonal antibodies CD20 is an integral protein specific to B-lymphocytes and therefore an attractive target for B-cell malignancies and B-cell-mediated autoimmune diseases. It is a transmembrane receptor, although the natural ligand is not known yet, and its physiological role is not completely understood. ${ }^{9}$ It is suspected that it is involved in the regulation of B-cell activation and proliferation and that it is crucial for B-cell immune response..$^{10}$ Depending on the targeted epitope, CD20 antibodies can have different affinities and induce varying immune responses.

There are two types of CD20 monoclonal antibodies (Table 1). Type I antibodies such as rituximab and ofatumumab bind to CD20 and induce a quick redistribution of the antibody-antigen complex into a lipid raft. ${ }^{11}$ This complex only leads to weak direct cell death or accordingly apoptosis, but strong complement-dependent cytotoxicity (CDC) by recruiting C1q. ${ }^{12}$ Ofatumumab has a particular high affinity and powerful $\mathrm{CDC}$ activity due to a distinct binding site at the CD20 transmembrane protein, which differs from rituximab's binding region. ${ }^{13}$ In contrast, type II antibodies such as obinutuzumab do not localize the antibody-antigen complex into lipid rafts and therefore induce only very weak CDC that is 10 - to 100 -fold weaker than that with rituximab or ofatumumab. ${ }^{14}$ However, reduced Fc $\gamma$ RIIb-mediated CD20

Table I Type I and type II antibodies

\begin{tabular}{ll}
\hline Type I & Type II \\
\hline Rituximab, ofatumumab & Obinutuzumab \\
Strong CDC & Weak CDC \\
Weak direct cell death & Strong direct cell death \\
Moderate ADCC & Strong ADCC \\
Moderate ADCP & Strong ADCP \\
\hline
\end{tabular}

Note: Data from Cartron, ${ }^{7}$ Goede et al $^{8}$ and Klein et al. ${ }^{59}$

Abbreviations: ADCC, antibody-dependent cell-mediated cytotoxicity; ADCP, antibody-dependent cellular phagocytosis; CDC, complement-dependent cytotoxicity. internalization increases the capacity to bind and activate natural killer (NK) cells and subsequent immune effector function. ${ }^{15,16}$ Additionally, obinutuzumab causes cell death via homotypic aggregation, meaning the aggregation of malignant B-cells by antibodies and subsequent nonapoptotic cell death without the involvement of immune effector cells. ${ }^{17}$ It was suggested that obinutuzumab binding leads to activation of a family of Rho kinases that are involved in B-cell receptor (BCR) activation as well as cytoskeletal rearrangements. ${ }^{18}$ Preclinical observations confirmed that obinutuzumab induced rapid relocalization of actin filaments together with cell-surface antigens toward cell-cell junctions and thereby also activated lysosomes. These lysosomes play an important role in triggering caspase-independent cell death. ${ }^{19,20}$ In particular, it was shown that upon binding of type II antibodies onto CLL cells, lysosomes release various enzymes, including cathepsin B, and thereby induce cell death independently of caspases and without involvement of B-cell lymphoma-2 (Bcl-2). ${ }^{21}$ This mechanism is not entirely understood but has been previously described for other antigens and is of particular interest in CLL patients who often present with impaired immunity. ${ }^{21}$ Furthermore, obinutuzumab mediates increased binding and activation of NK cells particularly via Fc $\gamma$ RIIIa, which ultimately leads to enhanced antibody-dependent cell-mediated cytotoxicity (ADCC) and antibody-dependent cellular phagocytosis (ADCP). ${ }^{16}$

\section{Modification of CD20 monoclonal antibodies}

In order to achieve the distinct characteristics of obinutuzumab, various modifications of the antibody structure were performed. Generally, antibody activity can be manipulated by retargeting to a different CD20 epitope. For instance, ofatumumab binds to a different epitope than rituximab and by that induces increased CDC. ${ }^{13}$ Likewise, modification of the Fc region of the antibody can enhance immune effector functions; eg, ocaratuzumab, a type I antibody currently under development, has nine amino acid changes in its Fc region when compared to rituximab, which led to an up to 20-fold increased CD20 affinity and 6-fold increased ADCC. ${ }^{22}$

In comparison to rituximab, obinutuzumab binds to a more exposed epitope of CD20, which though overlaps with rituximab's binding region. ${ }^{23}$ However, the antibody has a completely different spatial orientation of binding, which is achieved by substitution of the amino acid leucine by valine at the antibody's hinge region. ${ }^{24}$ This leads to a $30^{\circ}$ wider elbow angle of obinutuzumab at the CD20 antigen. In vitro, the different spatial arrangement leads to more direct cell 
death induction, probably due to higher affinity and more flexibility of binding. ${ }^{17}$

Antibody affinity to the Fc $\gamma$ RIII fragment is particularly important, as this mediates the interaction with immune effector cells. The affinity can be modified by changing the structure of oligosaccharides that are attached to the antibody's Fc fragment, causing a more intense interaction with neutrophil granulocytes as well as NK cells. This process is called glycoengineering: in an experimental setting, obinutuzumab was initially designed by removal of one fucose from the glycan tree that is attached to the amino acid asparagine $297 . .^{25,26}$ This led to an increased affinity to

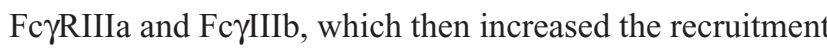
of FcyRIII expressing effector cells (ie, neutrophil granulocytes, NK cells and macrophages), and more intense signaling was observed. ${ }^{14}$ ADCC was increased in comparison to fully fucosylated antibodies, while ADCP seemed to be comparable. In comparison to rituximab, NK degranulation was two- to fourfold more efficient with obinutuzumab. ${ }^{27}$ This unique process of glycoengineering was standardized by raising antibodies in Chinese hamster ovary (CHO) cells that constitutively overexpress the heavy and light chains of obinutuzumab together with $\beta-1,4-N$-acetylglucosaminyltransferase III and Golgi $\alpha$-mannosidase II, both of which reduce fucose and thereby remove fucose from the glycan tree of obinutuzumab. ${ }^{28}$

In summary, the modification of the hinge region as well as of the Fc-attached glycan tree led to the distinct functional characteristics of obinutuzumab, which differ from those of rituximab and ofatumumab (Figure 1).

\section{Preclinical data}

Prior to human use, obinutuzumab was tested in several preclinical models to further investigate its immunomodulatory capacities. Early experiments in human lymphoma xenograft models showed that obinutuzumab was able to achieve complete tumor regression in an aggressive diffuse large B-cell lymphoma (DLBCL) model using human SUDHL-4 cells, which were subcutaneously injected in severe combined immune deficiency (SCID) mice. ${ }^{17}$ In this model, rituximab only inhibited further tumor growth but failed to achieve regression. In a similar model with more aggressive, rituximab-refractory DLBCL, obinutuzumab was able to induce tumor growth control in a dose-dependent manner.

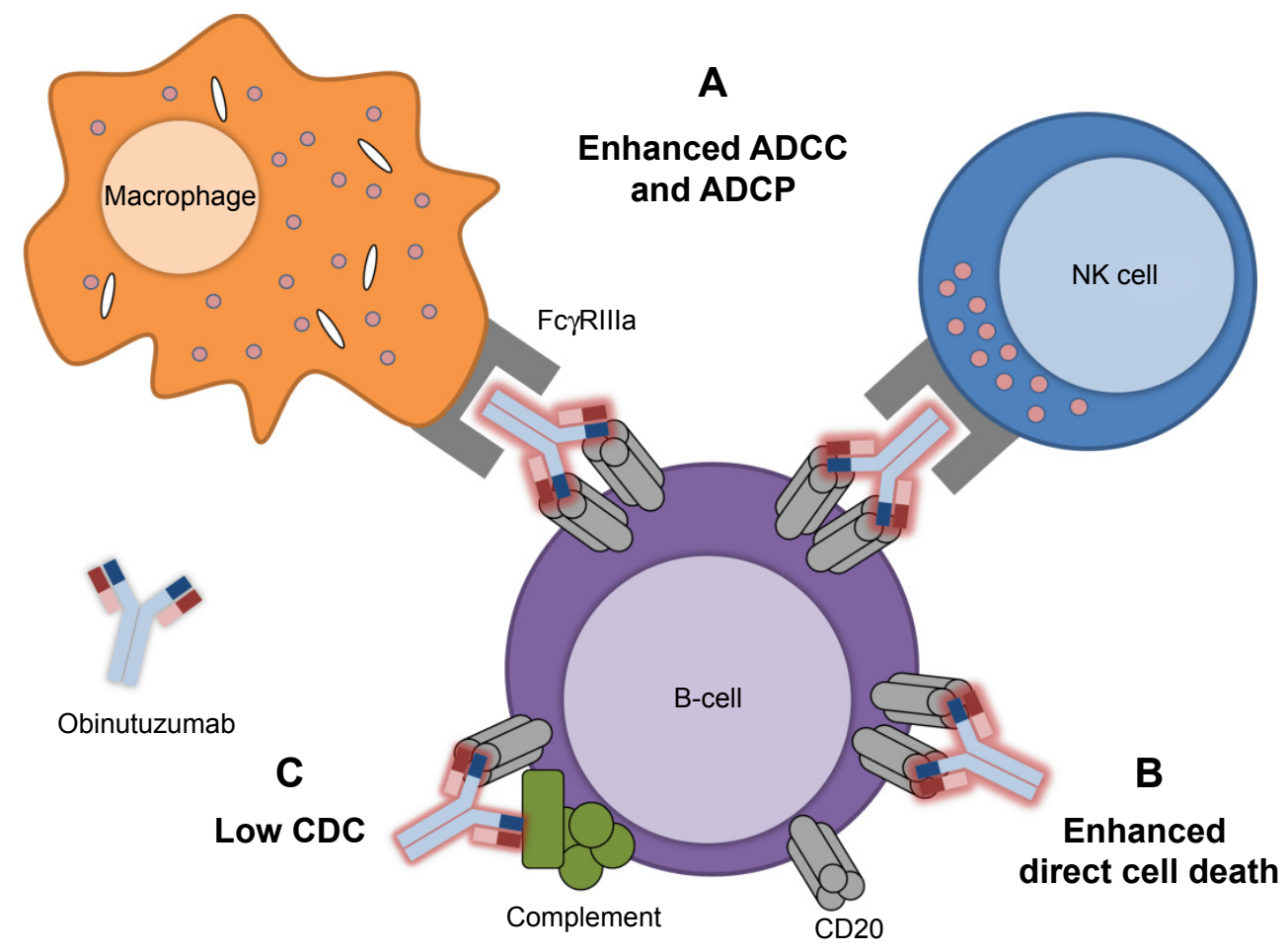

Figure I Mechanism of action of obinutuzumab.

Notes: (A) Modification of the glycan tree structure at the Fc fragment of obinutuzumab leads to an increased affinity to Fc $\gamma$ RIII and thereby ADCC via NK cells as well as ADCP via macrophages is intensified. (B) Direct cell death is not dependent on immune effector cells and is mediated via induction of apoptotic pathways upon antibody binding as well as homotypic aggregation, ie, aggregation of malignant B-cells with subsequent nonapoptotic cell death without involvement of immune effector cells. (C) Obinutuzumab only induces weak CDC in comparison to type I antibodies such as rituximab and ofatumumab, mainly due to the lack of lipid raft formation upon antibody binding to CD20.

Abbreviations: ADCC, antibody-dependent cell-mediated cytotoxicity; ADCP, antibody-dependent cellular phagocytosis; CDC, complement-dependent cytotoxicity; NK, natural killer. 
Similar outcomes were reported from an experiment using a model for advanced mantle cell lymphoma with Z138 MCL cells in SCID mice, where obinutuzumab showed superior efficacy with regard to OS when compared to rituximab. ${ }^{29}$

Mossner et $\mathrm{al}^{17}$ also investigated the extent of B-cell depletion in cynomolgus monkeys treated with different doses of rituximab and obinutuzumab. Both antibodies achieved highly efficient depletion of B-lymphocytes from peripheral blood (PB); however, obinutuzumab also efficiently depleted B-cells in the spleen and in lymph nodes. Memory B-cells and long-lived plasma cells were spared from depletion, implicating that humoral immunity and memory remained intact. ${ }^{17}$ In the setting of CLL, obinutuzumab also induced greater levels of B-cell depletion than rituximab in whole blood taken from healthy donors as well as CLL patients. ${ }^{30}$

Overall, accumulating evidence from preclinical experiments suggested that obinutuzumab had promising activity in various models of NHL, including DLBCL, MCL and CLL, with superiority over rituximab.

\section{Clinical data}

\section{Efficacy}

Obinutuzumab was first tested in the Phase I/II GAUGUIN trial, which enrolled 33 patients with relapsed/refractory CLL. ${ }^{31}$ The trial was separated into a dose escalation phase and a fixed dose phase. Patients initially received 400-1,200 mg of obinutuzumab in Phase I and a fixed dose of $1,000 \mathrm{mg}$ in Phase II. An overall response rate (ORR) of $62 \%$ was reported in the first phase, but only of $15 \%$ in the second phase; no complete remissions (CRs) were observed. The striking difference in response rates between the trial phases was attributed to an imbalance in tumor burden between the two patient cohorts.

Further investigation on obinutuzumab dosage was done in the GAGE trial, which compared 1,000 vs 2,000 mg obinutuzumab (100 mg intravenous [IV] day 1, 900 [1,900] mg day 2 , $1,000[2,000] \mathrm{mg}$ days 8 and 15 in cycle $1 ; 1,000$ [2,000] $\mathrm{mg}$ day 1 over 7 further cycles). ${ }^{32}$ Eighty treatment-naïve patients were enrolled for this Phase II study. With regard to the primary end point, ORR was higher in the 2,000 mg cohort (67\% vs 49\%) as well as CR (20\% vs 5\%). Despite this dose effect on response, no difference in PFS was observed.

The GALTON trial investigated the combination of obinutuzumab with a backbone chemotherapy of investigators' choice in 41 treatment-naïve CLL patients. ${ }^{33}$ Obinutuzumab plus fludarabine and cyclophosphamide (FC-Ob) led to an ORR of $62 \%$, including $10 \%$ CR. A combination with bendamustine achieved an ORR of $90 \%$ with a CR rate of $20 \%$.
Notable adverse events with obinutuzumab were grade 3/4 neutropenia in $48 \%$ of the patients treated with $\mathrm{FC}-\mathrm{Ob}$ and $55 \%$ in the bendamustine plus obinutuzumab (B-Ob) arm. Infusion-related reactions (IRRs) occurred in $29 \%$ of FC-Ob and $10 \%$ of $\mathrm{B}-\mathrm{Ob}$ patients, but only during the administration of the first dose.

The pivotal CLL11 trial by the GCLLSG investigated obinutuzumab as frontline treatment in CLL patients with significant comorbidities, defined by a cumulative illness rating scale (CIRS) score of $>6$ points and/or a creatinine clearance of $<70 \mathrm{~mL} / \mathrm{min} .{ }^{34}$ At the time of study initiation, a standard of care for this group of patients was chlorambucil monotherapy. Thus, the trial compared three treatment modalities: chlorambucil alone (Clb-mono) vs rituximab plus chlorambucil (R-Clb) vs obinutuzumab plus chlorambucil (Ob-Clb). Obinutuzumab was initially administered at $1,000 \mathrm{mg}$ on days 1,8 and 15 in the first cycle and subsequently at $1,000 \mathrm{mg}$ on day 1 of the following five cycles. For better tolerability, a protocol amendment allowed a separation of the first obinutuzumab dose into $100 \mathrm{mg}$ and $900 \mathrm{mg}$ on days 1 and 2. Rituximab was administered at $375 \mathrm{mg} / \mathrm{m}^{2}$ on day 1 of the first cycle and $500 \mathrm{mg} / \mathrm{m}^{2}$ on day 1 of the subsequent five cycles. Chlorambucil was administered at $0.5 \mathrm{mg} / \mathrm{kg}$ on days 1 and 15 in each cycle.

The trial was divided into two stages: the aim of the first stage was to analyze whether addition of obinutuzumab or rituximab has any benefit in comparison to chlorambucil monotherapy. A total of 118 patients received Clb-mono, 238 received $\mathrm{Ob}-\mathrm{Clb}$ and 233 received $\mathrm{R}-\mathrm{Clb}$. ORR in the Clb-mono arm was $31.4 \%$ (0\% CR) vs $77.3 \%(22.3 \% \mathrm{CR})$ in the $\mathrm{Ob}-\mathrm{Clb}$ arm and $65.7 \%(7.3 \% \mathrm{CR})$ in the $\mathrm{R}-\mathrm{Clb}$ arm. No patient achieved minimal residual disease (MRD) negativity (assessed by PCR) in the Clb-mono arm, in contrast to $\mathrm{Ob}-\mathrm{Clb}$ (17\% in bone marrow [BM], 31.1\% in PB) and R-Clb $(2.8 \%$ in $\mathrm{BM}, 2.0 \%$ in $\mathrm{PB}$ ). To date, $\mathrm{Ob}-\mathrm{Clb}$ achieved the highest rate of MRD negativity in this patient population. After a median observation time of 23 months, median PFS in the Clb-mono group (11.1 months) was significantly shorter than in the R-Clb (15.4 months) as well as the Ob-Clb arm (29.2 months).

More patients were randomized into stage 2 of the trial, which aimed to compare R-Clb with Ob-Clb. A total of 333 patients received $\mathrm{Ob}-\mathrm{Clb}$ and 330 received $\mathrm{R}-\mathrm{Clb}$. ORR was significantly improved by obinutuzumab when compared to rituximab (78.4\% vs $65.1 \%)$, including a higher $\mathrm{CR}$ rate (20.7\% vs 7\%). Likewise, MRD negativity rate was also higher in the $\mathrm{Ob}-\mathrm{Clb}$ group $(19.5 \%$ vs $2.6 \%$ in $\mathrm{BM}$ and $37.7 \%$ vs $3.3 \%$ in $\mathrm{PB})$. After a median observation time of 
18.7 months, patients in the $\mathrm{Ob}-\mathrm{Clb}$ group had a significantly longer PFS (26.7 months vs 15.2 months). While this did not translate into an OS benefit (hazard ratio [HR] for death 0.66 ; $P=0.08$ ), addition of obinutuzumab did in fact increase OS when compared to $\mathrm{Clb}$-mono (HR $0.41 ; P=0.002$ ).

Meanwhile, updated results of the CLL11 study with longer observation time have been published and confirmed the initial findings outlined earlier. ${ }^{35}$ Importantly, these results showed a significant and clinically meaningful extension of time to next treatment (TTNT) from 38.2 months in the R-Clb group to 51.1 months in the $\mathrm{Ob}-\mathrm{Clb}$ group (HR 0.57, 95\% CI 0.44-0.74, $P<0.0001)$.

Obinutuzumab has also been tested in other B-cell NHL, including DLBCL and follicular lymphoma (FL). The GOYA trial compared rituximab plus cyclophosphamide, hydroxydaunorubicin, oncovin, prednisone (CHOP) (R-CHOP) with obinutuzumab plus CHOP (G-CHOP) in 1,418 patients with DLBCL. The primary end point was investigator-assessed PFS. However, the end point was not met as no significant PFS difference between R-CHOP and G-CHOP was detected (3y-PFS was $66 \%$ for R-CHOP and 69\% for G-CHOP). ${ }^{36}$ Recently released data on obinutuzumab in FL are more promising: the GALLIUM trial recently showed that induction chemoimmunotherapy with CHOP, CVP or bendamustine followed by maintenance is more effective when obinutuzumab instead of rituximab is used as the antibody. Of the 1,202 patients with FL, G-based therapy yielded a median investigator-assessed $3 y-P F S$ of $80 \%$ vs $73 \%$ in those receiving R-based therapy. This was a clinically meaningful reduction of risk of progression by $34 \%$. Adverse events were comparable to those previously described in CLL11 and were mostly related to more frequent cytopenias and IRRs. ${ }^{37}$

Taken together, the additional benefit of obinutuzumab compared to rituximab has been substantiated in several Phase II and two large Phase III clinical trials. Based on the results of the CLL11 trial, obinutuzumab was approved by the authorities for first-line treatment in combination with chlorambucil in previously untreated CLL patients with comorbidities.

\section{Safety and tolerability}

In CLL11, the most common severe toxicities (grade 3 and 4) associated with obinutuzumab were IRRs (21\%), neutropenia (34\%) and thrombocytopenia (12\%). From the first trial onward, an increased rate of IRRs was described particularly during first administration of obinutuzumab. In CLL1 1, high-grade IRRs occurred in $21 \%$ of the patients receiving obinutuzumab in stage 1 and $20 \%$ in stage 2 , while rituximab only induced high-grade IRRs in $4 \%$ of the patients. It was suggested that the quick clearance of the PB from lymphocytes observed with obinutuzumab (Figure 2) might trigger a cytokine release, which clinically leads to IRR. The source of IRR-driving cytokines (mainly IL-6) is unknown; however, alternatively immune effector cells could be recruited by obinutuzumab as part of ADCC. Severe IRRs upon obinutuzumab administration occurred only during the first infusion, and different measurements were taken in order to manage IRRs in the clinical setting. First, the initial dose of 1,000 $\mathrm{mg}$ was administered over 2 days (100 $\mathrm{mg}$ on day 1 and $900 \mathrm{mg}$ on day 2) and additionally a prophylaxis with acetaminophen, an antihistamine and $100 \mathrm{mg}$ of IV prednisone was given before the first dose of obinutuzumab. This strategy allowed good management of IRR also in subsequent clinical trials using obinutuzumab.

Several reasons for the increased occurrence of IRRs have been discussed. Obinutuzumab has a high affinity for FcyRIII (the "Function of CD20 monoclonal antibodies" section), leading to strong binding to CD20 and stronger Fc $\gamma R$ activation and effector cell recruitment particularly in the PB. This mediates a strong cytokine release, which clinically became manifest particularly in patients with high peripheral CLL cell counts. ${ }^{38}$ Moreover, Trisomy 12, which is known to be associated with higher levels of CD20 expression, was found to be a risk factor for the occurrence of IRRs. ${ }^{39}$ Cytokine profiling showed that levels of TNF- $\alpha$, IFN- $\gamma$ as well as IL- 6 and IL-8 are significantly increased after the first obinutuzumab infusion, while the number of circulating B-lymphocytes rapidly fell. ${ }^{38}$

Cytopenias were also more frequent under obinutuzumab. In particular, grade 3 neutropenias occurred more frequently during $\mathrm{Ob}-\mathrm{Clb}$ when compared to $\mathrm{Clb}$-mono (35\% vs $16 \%$ ). Fortunately, the rate of grade 3-4 infections was not higher with Ob-Clb than with Clb-mono (11\% vs 14\%). Comparison of $\mathrm{Ob}-\mathrm{Clb}$ and $\mathrm{R}-\mathrm{Clb}$ in stage 2 of the trial revealed a trend toward higher rates of neutropenia and thrombocytopenia with obinutuzumab (33\% vs $28 \%$ and $28 \%$ vs $11 \%$, respectively). Again, rates of infections were comparable (12\% vs 14\%). Preclinical models have suggested that the reason for the more pronounced neutropenia is the enhanced recruitment and consumption of neutrophils in the course of obinutuzumab-mediated ADCP. ${ }^{27}$

In summary, therapy of CLL with obinutuzumab is associated with more frequent IRRs as well as more pronounced cytopenias. So far, both of these adverse reactions were well manageable in clinical trials and did not interfere with therapeutic efficacy. 


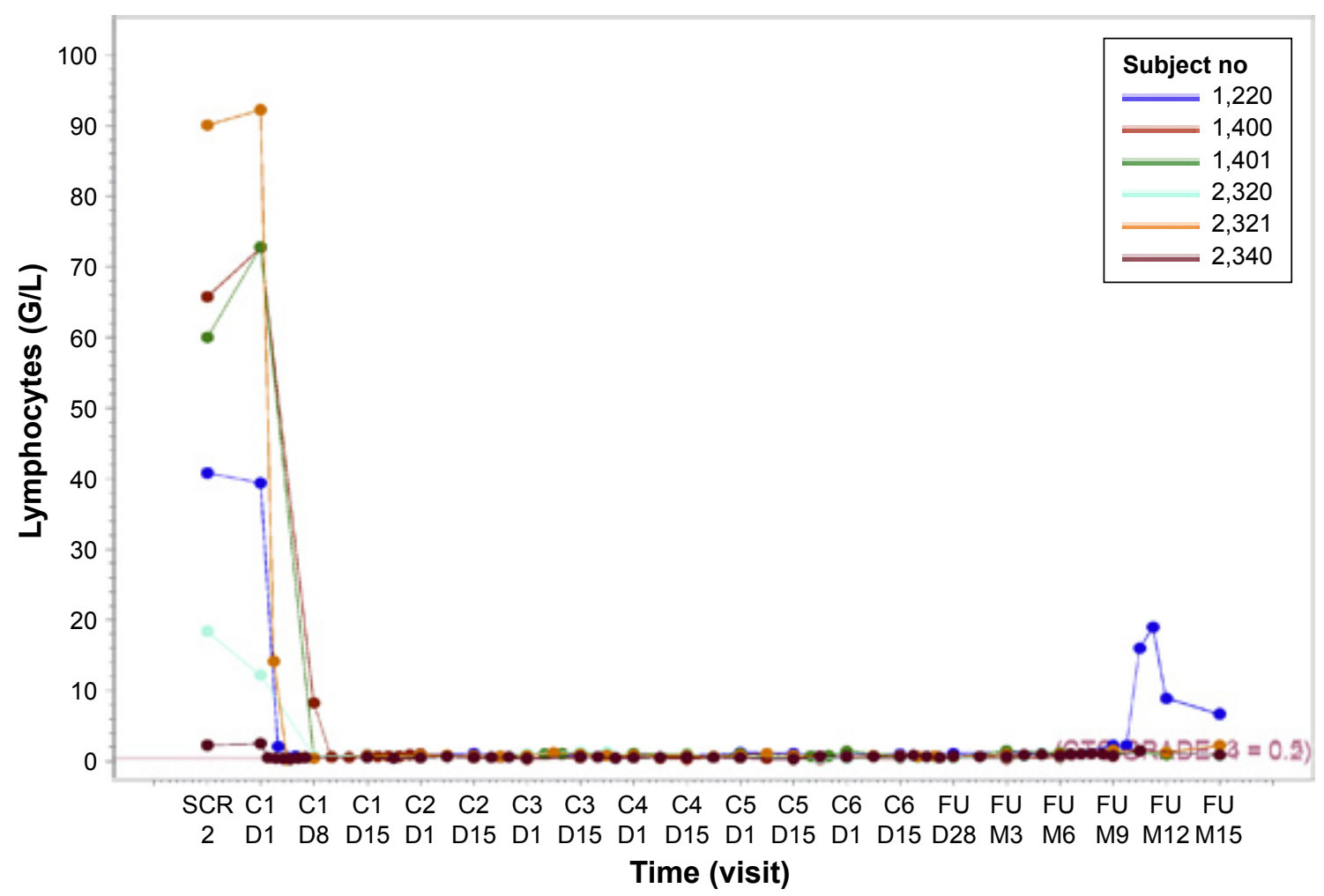

Figure 2 Peripheral lymphocyte count clearance.

Notes: Peripheral blood lymphocyte counts during and after chemoimmunotherapy with obinutuzumab plus chlorambucil. Reproduced from Goede V, Fischer K, Busch R, et al. Chemoimmunotherapy with GAIOI plus chlorambucil in patients with chronic lymphocytic leukemia and comorbidity: results of the CLLII (BO2I004) safety run-in. Leukemia. 2013;27(5): $1172-1174 .{ }^{60}$

Abbreviations: SCR, screening; C, cycle; FU, follow-up; D, day; M, month.

\section{Outlook: new indications, new combinations and new challenges}

Rituximab has become an integral part of first-line treatment for most CLL patients. In order to find out whether a nextgeneration CD20 antibody such as obinutuzumab is superior in the first-line setting, further clinical trials comparing FCR/BR with FC-Ob/B-Ob would be desirable. Currently, there are several ongoing Phase II trials that explore the efficacy and tolerability of FC-Ob/B-Ob in treatment-naïve as well as pretreated patients (NCT02071225, NCT02320487 and NCT02320383). Moreover, the GREEN trial, a Phase IIIb multicenter, open-label trial, currently explores the safety and efficacy of obinutuzumab alone or in combination with chemotherapy (bendamustine, chlorambucil or cyclophosphamide/fludarabine) in fit and unfit untreated or pretreated CLL patients (NCT01905943). So far, data on first-line B-Ob have shown an ORR of 78.5\% (CR 32.3\%) and an MRD negativity rate of $58.9 \%$ in $\mathrm{PB}$ or accordingly $27.8 \%$ in BM. Overall, AEs were comparable to $\mathrm{Ob}-\mathrm{Clb}$ with IRRs in $66.7 \%$ of patients $(15.2 \% \geq$ grade 3 , none fatal), neutropenia (10.8\%), pyrexia (7.6\%) and febrile neutropenia (7\%). Tumor lysis syndrome was observed in $5.1 \%$ of the patients. B-Ob was well tolerated and manageable, making it a possible future alternative for treatment of fit and maybe unfit CLL patients. ${ }^{40}$

\section{New combinational strategies}

The introduction of inhibitors of the BCR signaling pathway, such as ibrutinib, a Bruton's tyrosine kinase (BTK) inhibitor, or idelalisib, a PI-3 kinase inhibitor, as well as venetoclax, a Bcl-2 inhibitor, has enlarged the therapeutic landscape in CLL. ${ }^{41}$ A key question is whether combinations of small molecules with CD20 antibodies provide additional benefit or whether these new compounds are sufficiently effective as monotherapies. Ibrutinib has shown superior activity with durable remissions in treatment-naïve as well as relapsed/refractory CLL patients, including patients with unfavorable mutations such as deletion of $17 \mathrm{p}$ or patients with comorbidities. ${ }^{42}$ However, preclinical research has indicated that ibrutinib might inhibit ADCC by blocking IL-2-inducible tyrosine kinase, a member of the BTK family, which is needed for activation of NK cells. ${ }^{43}$ Therefore, it was suggested that CD20 antibodies relying on ADCC might be impaired by ibrutinib. Burger et $\mathrm{al}^{44}$ investigated a 
combination of ibrutinib plus rituximab in a Phase II trial and reported promising efficacy with an 18-month PFS of 78\% ( $72 \%$ in patients with del(17p), ORR and CR were $95 \%$ and $8 \%$, respectively). A randomized controlled trial comparing ibrutinib with ibrutinib plus rituximab was subsequently started (NCT02007044). These preclinical findings were not observed in clinical trials, suggesting that rituximab's activity particularly relies on $\mathrm{CDC}$ rather than ADCC and therefore was not impaired by ibrutinib.

However, a combination of ibrutinib and obinutuzumab, which mediates its cytotoxic activity essentially via ADCC, is also currently investigated in a Phase II trial by the GCLLSG (CLL2-BIG trial, NCT02345863).

Idelalisib was licensed based on a Phase III trial comparing idelalisib plus rituximab vs rituximab alone in 220 frail, elderly patients with relapsed CLL. ${ }^{45}$ Subsequent approaches are investigating a combination of idelalisib plus obinutuzumab, for instance in the CLL2-BCG trial of the GCLLSG (NCT02445131).

Finally, obinutuzumab is currently also being tested in combination with the Bcl-2 inhibitor venetoclax. The CLL14 study, successor trial of CLL11, compares the standard of care for comorbid patients (Ob-Clb) with obinutuzumab plus venetoclax (NCT02242942). First results of the safety run-in phase of the trial showed good tolerability and response to this combination. ${ }^{46}$ Likewise, the CLL2-BAG trial of the GCLLSG compares a similar combination after bendamustine debulking in treatment-naïve as well as pretreated CLL patients (NCT02401503).

Importantly, while combinational strategies are likely to further enhance therapeutic efficacy, further data on safety and tolerability of such new regimes are warranted before they can be applied in clinical practice.

\section{Rituximab-refractory disease}

Resistance to type I CD20 antibodies such as rituximab is rare and incompletely understood. In some rituximabrefractory CLL patients, reduced expression of CD20 has been suggested as an escape mechanism of the lymphoma cells. ${ }^{19,47}$ The protein can be removed from cell surface by phagocytic cells ("shaving") or internalized via endocytosis into lysosomes. ${ }^{48-50}$

Moreover, the interaction between the Fc fragment of the CD20 antibody and FcyR on leukocytes is essential for ADCC and ADCP. Different polymorphisms in Fc $\gamma \mathrm{R}$ can either increase or decrease cytotoxicity of rituximab and are therefore a possible mechanism of resistance. ${ }^{51}$ Likewise, changes in patient's physiology such as impaired cellular immunity due to prior chemotherapies or an increased degradation of monoclonal antibodies can reduce response to rituximab.

In Phase II trials with rituximab-refractory NHL (including CLL), obinutuzumab has shown single-agent activity. ${ }^{52,53}$ In the GAUGUIN trial, patients with relapsed or refractory DLBCL receiving obinutuzumab monotherapy achieved an ORR of up to $37 \%$ and patients with relapsed or refractory mantle cell lymphoma achieved an ORR of $20 \% .^{31}$ A median duration of response of 9.8 months was observed, which was notably sustained in rituximab-refractory patients, who had a response duration of 9 months.

Moreover, combination of obinutuzumab with bendamustine was very effective in the Phase III GADOLIN trial, ${ }^{54}$ which compared the efficacy of bendamustine monotherapy (B) vs B-Ob in rituximab refractory indolent NHL. ORR did not differ between the two groups (63\% vs 69\%), but after a median follow-up time of 21.9 months, median PFS was 29.2 months in the B-Ob group vs 14 months in the B group. The exact mechanism by which type II CD20 antibodies may overcome rituximab resistance is not understood. However, it is possible that this is related to the different targeted epitopes as well as the more efficient recruitment of effector cells. ${ }^{55}$

\section{Summary and conclusion}

Obinutuzumab is the first licensed glycoengineered type II CD20 antibody that compared to rituximab induces more enhanced cell death via ADCC and ADCP. Its high efficacy was first confirmed in preclinical models, in which modification of the antibody structure as well as the attached glycan tree induced a more pronounced B-cell depletion. Subsequent clinical trials confirmed the high efficacy of obinutuzumab in CLL patients. The CLL11 trial demonstrated that obinutuzumab can induce considerable rates of MRD negativity in comorbid patients with durable remissions. Therefore, $\mathrm{Ob}-\mathrm{Clb}$ is a current standard of care for previously untreated CLL patients with comorbidities.

The therapeutic landscape in CLL is currently very dynamic and new therapeutic strategies and schedules are constantly being investigated. The development of potent single-agent regimes such as ibrutinib and combinations of idelalisib plus rituximab also suggests a possible efficacy of combinations of small molecules with obinutuzumab. To date, the benefit of such strategies is not completely established. Combinations of obinutuzumab with one or even two small molecules, eg, venetoclax plus obinutuzumab (NCT02242942), ibrutinib plus obinutuzumab (NCT02345863) or ibrutinib plus venetoclax plus 
obinutuzumab (CLL13 trial of the GCLLSG, recruiting planned in Q4/2016), are possible chemotherapy-free regimens that might challenge chemoimmunotherapies in the first- and second-line setting.

The clinical advantage of such combinational strategies and particularly their superiority over conventional therapies needs to be rigorously tested as many health systems could be overburdened by the additional costs of such regimens, which jeopardizes the sustainability and feasibility of such novel therapies. While $\mathrm{Ob}-\mathrm{Clb}$ seems to be cost-effective overall in the long run, ${ }^{56}$ other obinutuzumab combinations still need to be tested for efficacy as well as feasibility in randomized controlled trials.

\section{Disclosure}

OA has received honoraria and travel grants from Roche and travel grants from Gilead. KF and AE have received travel grants from Roche. NP has received honoraria from Novartis and travel grants from Jazz Pharmaceuticals and Celgene. VG has received consultancy, honoraria and travel grants from Roche; consultancy from Gilead; consultancy and travel grants from Janssen; consultancy and honoraria from GlaxoSmithKline and Mundipharma and honoraria from Bristol Myer Squibb. MH has received consultancy and honoraria from AbbVIe, Mundipharma, GlaxoSmithKline, Gilead and Celgene; consultancy, honoraria and speakers bureau from Janssen; consultancy and speakers bureau from Pharmacyclics and consultancy, research funding and speakers bureau from Roche. The authors report no other conflicts of interest in this work.

\section{References}

1. Fischer K, Bahlo J, Fink AM, et al. Long-term remissions after FCR chemoimmunotherapy in previously untreated patients with CLL: updated results of the CLL8 trial. Blood. 2016;127(2):208-215.

2. Hallek M, Fischer K, Fingerle-Rowson G, et al; International Group of Investigators; German Chronic Lymphocytic Leukaemia Study Group. Addition of rituximab to fludarabine and cyclophosphamide in patients with chronic lymphocytic leukaemia: a randomised, open-label, phase 3 trial. Lancet. 2010;376(9747):1164-1174.

3. Eichhorst B, Fink A-M, Bahlo J, et al. First-line chemoimmunotherapy with bendamustine and rituximab versus fludarabine, cyclophosphamide, and rituximab in patients with advanced chronic lymphocytic leukaemia (CLL10): an international, open-label, randomised, phase 3, non-inferiority trial. Lancet Oncol. 2016;17(7):928-942.

4. O'Brien SM, Kantarjian H, Thomas DA, et al. Rituximab dose-escalation trial in chronic lymphocytic leukemia. J Clin Oncol. 2001;19(8): 2165-2170.

5. Thompson PA, Tam CS, O'Brien SM, et al. Fludarabine, cyclophosphamide, and rituximab treatment achieves long-term disease-free survival in IGHV-mutated chronic lymphocytic leukemia. Blood. 2016;127(3): 303-309.

6. Chaudhry M, Cheson BD. What is the status of novel anti-CD20 antibodies for chronic lymphocytic leukemia and are they set to leave rituximab in the shadows? Expert Rev Hematol. 2015;8(6):733-742.
7. Gagez AL, Cartron G. Obinutuzumab: a new class of anti-CD20 monoclonal antibody. Curr Opin Oncol. 2014;26(5):484-491.

8. Goede V, Klein C, Stilgenbauer S. Obinutuzumab (GA101) for the treatment of chronic lymphocytic leukemia and other B-cell non-Hodgkin's lymphomas: a glycoengineered type II CD20 antibody. Oncol Res Treat. 2015;38(4):185-192.

9. Cragg MS, Walshe CA, Ivanov AO, Glennie MJ. The biology of CD20 and its potential as a target for mAb therapy. Curr Dir Autoimmun. 2005; 8:140-174.

10. Woyach JA, Johnson AJ, Byrd JC. The B-cell receptor signaling pathway as a therapeutic target in CLL. Blood. 2012;120(6):1175-1184.

11. Chan HT, Hughes D, French RR, et al. CD20-induced lymphoma cell death is independent of both caspases and its redistribution into triton X-100 insoluble membrane rafts. Cancer Res. 2003;63(17): 5480-5489.

12. Cragg MS, Morgan SM, Chan HT, et al. Complement-mediated lysis by anti-CD20 mAb correlates with segregation into lipid rafts. Blood. 2003;101(3):1045-1052.

13. Teeling JL, Mackus WJ, Wiegman LJ, et al. The biological activity of human CD20 monoclonal antibodies is linked to unique epitopes on CD20. J Immunol. 2006;177(1):362-371.

14. Herter S, Herting F, Mundigl O, et al. Preclinical activity of the type II CD20 antibody GA101 (obinutuzumab) compared with rituximab and ofatumumab in vitro and in xenograft models. Mol Cancer Ther. 2013;12(10): 2031-2042.

15. Kern DJ, James BR, Blackwell S, Gassner C, Klein C, Weiner GJ. GA101 induces NK-cell activation and antibody-dependent cellular cytotoxicity more effectively than rituximab when complement is present. Leuk Lymphoma. 2013;54(11):2500-2505.

16. Lim SH, Vaughan AT, Ashton-Key M, et al. Fc gamma receptor IIb on target B cells promotes rituximab internalization and reduces clinical efficacy. Blood. 2011;118(9):2530-2540.

17. Mossner E, Brunker P, Moser S, et al. Increasing the efficacy of CD20 antibody therapy through the engineering of a new type II anti-CD20 antibody with enhanced direct and immune effector cell-mediated B-cell cytotoxicity. Blood. 2010;115(22):4393-4402.

18. Arana E, Vehlow A, Harwood NE, et al. Activation of the small GTPase Rac2 via the B cell receptor regulates B cell adhesion and immunological-synapse formation. Immunity. 2008;28(1):88-99.

19. Alduaij W, Ivanov A, Honeychurch J, et al. Novel type II anti-CD20 monoclonal antibody (GA101) evokes homotypic adhesion and actindependent, lysosome-mediated cell death in B-cell malignancies. Blood. 2011;117(17):4519-4529.

20. Dalle S, Reslan L, Besseyre de Horts T, et al. Preclinical studies on the mechanism of action and the anti-lymphoma activity of the novel anti-CD20 antibody GA101. Mol Cancer Ther. 2011;10(1):178-185.

21. Ivanov A, Beers SA, Walshe CA, et al. Monoclonal antibodies directed to CD20 and HLA-DR can elicit homotypic adhesion followed by lysosome-mediated cell death in human lymphoma and leukemia cells. J Clin Invest. 2009;119(8):2143-2159.

22. Cheney CM, Stephens DM, Mo X, et al. Ocaratuzumab, an Fcengineered antibody demonstrates enhanced antibody-dependent cellmediated cytotoxicity in chronic lymphocytic leukemia. MAbs. 2014; 6(3):749-755.

23. Niederfellner G, Lammens A, Mundigl O, et al. Epitope characterization and crystal structure of GA101 provide insights into the molecular basis for type I/II distinction of CD20 antibodies. Blood. 2011;118(2):358-367.

24. Cartron G, Watier H, Golay J, Solal-Celigny P. From the bench to the bedside: ways to improve rituximab efficacy. Blood. 2004;104(9): 2635-2642.

25. Ferrara C, Grau S, Jager C, et al. Unique carbohydrate-carbohydrate interactions are required for high affinity binding between FcgammaRIII and antibodies lacking core fucose. Proc Natl Acad Sci U S A. 2011; 108(31):12669-12674.

26. Ferrara C, Stuart F, Sondermann P, Brunker P, Umana P. The carbohydrate at FcgammaRIIIa Asn-162. An element required for high affinity binding to non-fucosylated $\mathrm{IgG}$ glycoforms. $J$ Biol Chem. 2006;281(8):5032-5036. 
27. Golay J, Da Roit F, Bologna L, et al. Glycoengineered CD20 antibody obinutuzumab activates neutrophils and mediates phagocytosis through CD16B more efficiently than rituximab. Blood. 2013;122(20): 3482-3491.

28. Ferrara C, Brunker P, Suter T, Moser S, Puntener U, Umana P. Modulation of therapeutic antibody effector functions by glycosylation engineering: influence of Golgi enzyme localization domain and co-expression of heterologous beta 1, 4-N-acetylglucosaminyltransferase III and Golgi alpha-mannosidase II. Biotechnol Bioeng. 2006;93(5):851-861.

29. Herting F, Friess T, Bader S, et al. Enhanced anti-tumor activity of the glycoengineered type II CD20 antibody obinutuzumab (GA101) in combination with chemotherapy in xenograft models of human lymphoma. Leuk Lymphoma. 2014;55(9):2151-5160.

30. Patz M, Isaeva P, Forcob N, et al. Comparison of the in vitro effects of the anti-CD20 antibodies rituximab and GA101 on chronic lymphocytic leukaemia cells. Br J Haematol. 2011;152(3):295-306.

31. Cartron G, de Guibert S, Dilhuydy MS, et al. Obinutuzumab (GA101) in relapsed/refractory chronic lymphocytic leukemia: final data from the phase 1/2 GAUGUIN study. Blood. 2014;124(14):2196-2202.

32. Byrd JC, Flynn JM, Kipps TJ, et al. Randomized phase 2 study of obinutuzumab monotherapy in symptomatic, previously untreated chronic lymphocytic leukemia. Blood. 2016;127(1):79-86.

33. Brown JR, O'Brien S, Kingsley CD, et al. Obinutuzumab plus fludarabine/cyclophosphamide or bendamustine in the initial therapy of CLL patients: the phase 1b GALTON trial. Blood. 2015;125(18): 2779-2785.

34. Goede V, Fischer K, Busch R, et al. Obinutuzumab plus chlorambucil in patients with CLL and coexisting conditions. N Engl J Med. 2014; 370(12):1101-1110.

35. Goede V, Fischer K, Bosch F, et al. Updated Survival Analysis from the CLL11 Study: Obinutuzumab Versus Rituximab in Chemoimmunotherapy-Treated Patients with Chronic Lymphocytic Leukemia. ASH Annual Meeting; 2015; Orlando, FL.

36. Vitolo U, Trněný M, Belada D, et al. Obinutuzumab or Rituximab Plus CHOP in Patients with Previously Untreated Diffuse Large B-Cell Lymphoma: Final Results from an Open-Label, Randomized Phase 3 Study (GOYA) ASH Annual Meeting; 2016; San Diego, CA.

37. Marcus RE, Davies AJ, Ando K, et al. Obinutuzumab-Based Induction and Maintenance Prolongs Progression-Free Survival (PFS) in Patients with Previously Untreated Follicular Lymphoma: Primary Results of the Randomized Phase 3 GALLIUM Study. ASH Annual Meeting; 2016; San Diego, CA.

38. Freeman CLMF, Sehn LH, Dixon M, et al. Pattern of Cytokine Release in Patients with Chronic Lymphocytic Leukemia Treated with Obinutuzumab and Possible Relationship with Development of Infusion Related Reactions (IRR). San Francisco: ASH Annual Meeting; 2014.

39. Freeman CL, Dixon M, Houghton R, et al. Role of CD20 expression and other pre-treatment risk factors in the development of infusion-related reactions in patients with CLL treated with obinutuzumab. Leukemia 2016;30(8):1763-1766.

40. Stilgenbauer S, Ilhan O, Woszczyk D, et al. Safety and Efficacy of Obinutuzumab Plus Bendamustine in Previously Untreated Patients with Chronic Lymphocytic Leukemia: Subgroup Analysis of the Green Study. ASH Annual Meeting; 2015; Orlando, FL.

41. Cramer P, Hallek M, Eichhorst B. State-of-the-art treatment and novel agents in chronic lymphocytic leukemia. Oncol Res Treat. 2016;39(1-2): 25-32.

42. Byrd JC, Furman RR, Coutre SE, et al. Three-year follow-up of treatment-naive and previously treated patients with CLL and SLL receiving single-agent ibrutinib. Blood. 2015;125(16):2497-2506.

43. Kohrt HE, Sagiv-Barfi I, Rafiq S, et al. Ibrutinib antagonizes rituximabdependent NK cell-mediated cytotoxicity. Blood. 2014;123(12): 1957-1960.
44. Burger JA, Keating MJ, Wierda WG, et al. Safety and activity of ibrutinib plus rituximab for patients with high-risk chronic lymphocytic leukaemia: a single-arm, phase 2 study. Lancet Oncol. 2014;15(10): 1090-1099.

45. Furman RR, Sharman JP, Coutre SE, et al. Idelalisib and rituximab in relapsed chronic lymphocytic leukemia. N Engl J Med. 2014;370(11): 997-1007.

46. Fischer K, Fink AM, Bishop H, et al. Results of the Safety Run-in Phase of CLL14 (BO25323): A Prospective, Open-Label, Multicenter Randomized Phase III Trial to Compare the Efficacy and Safety of Obinutuzumab and Venetoclax (GDC-0199/ABT-199) with Obinutuzumab and Chlorambucil in Patients with Previously Untreated CLL and Coexisting Medical Conditions. Clinically Relevant Abstract. Orlando, Florida: ASH 2015; 2015

47. Davis TA, Czerwinski DK, Levy R. Therapy of B-cell lymphoma with anti-CD20 antibodies can result in the loss of CD20 antigen expression. Clin Cancer Res. 1999;5(3):611-615.

48. Beum PV, Kennedy AD, Williams ME, Lindorfer MA, Taylor RP. The shaving reaction: rituximab/CD20 complexes are removed from mantle cell lymphoma and chronic lymphocytic leukemia cells by THP-1 monocytes. J Immunol. 2006;176(4):2600-2609.

49. Hiraga J, Tomita A, Sugimoto T, et al. Down-regulation of CD20 expression in B-cell lymphoma cells after treatment with rituximab-containing combination chemotherapies: its prevalence and clinical significance. Blood. 2009;113(20):4885-4893.

50. Kennedy AD, Beum PV, Solga MD, et al. Rituximab infusion promotes rapid complement depletion and acute CD20 loss in chronic lymphocytic leukemia. J Immunol. 2004;172(5):3280-3288.

51. Cartron G, Dacheux L, Salles G, et al. Therapeutic activity of humanized anti-CD20 monoclonal antibody and polymorphism in IgG Fc receptor FcgammaRIIIa gene. Blood. 2002;99(3):754-758.

52. Morschhauser FA, Cartron G, Thieblemont C, et al. Obinutuzumab (GA101) monotherapy in relapsed/refractory diffuse large b-cell lymphoma or mantle-cell lymphoma: results from the phase II GAUGUIN study. J Clin Oncol. 2013;31(23):2912-2919.

53. Salles GA, Morschhauser F, Solal-Celigny P, et al. Obinutuzumab (GA101) in patients with relapsed/refractory indolent non-Hodgkin lymphoma: results from the phase II GAUGUIN study. J Clin Oncol. 2013; 31(23):2920-2926.

54. Sehn LH, Chua N, Mayer J, et al. Obinutuzumab plus bendamustine versus bendamustine monotherapy in patients with rituximab-refractory indolent non-Hodgkin lymphoma (GADOLIN): a randomised, controlled, open-label, multicentre, phase 3 trial. Lancet Oncology. 2016; 17(8):1081-1093.

55. Cartron G, Hourcade-Potelleret F, Morschhauser F, et al. Rationale for optimal obinutuzumab/GA101 dosing regimen in B-cell non-Hodgkin lymphoma. Haematologica. 2016;101(2):226-234.

56. Reyes C, Gazauskas G, Veentra D. Cost-effectiveness of obinutuzumab in previously untreated chronic lymphocytic leukemia. Madrid, Spain: ESMO Annual Meeting; 2014.

57. Jefferis R. Glycosylation as a strategy to improve antibody-based therapeutics. Nat Rev Drug Discov. 2009;8(3):226-234.

58. Illidge T, Klein C, Sehn LH, Davies A, Salles G, Cartron G. Obinutuzumab in hematologic malignancies: lessons learned to date. Cancer Treat Rev. 2015;41(9):784-792.

59. Klein C, Lammens A, Schafer W, et al. Epitope interactions of monoclonal antibodies targeting CD20 and their relationship to functional properties. MAbs. 2013;5(1):22-33.

60. Goede V,FischerK, Busch R, etal. Chemoimmunotherapy with GA101 plus chlorambucil in patients with chronic lymphocytic leukemia and comorbidity: results of the CLL11 (BO21004) safety run-in. Leukemia. 2013; 27(5):1172-1174 


\section{Publish your work in this journal}

Drug Design, Development and Therapy is an international, peerreviewed open-access journal that spans the spectrum of drug design and development through to clinical applications. Clinical outcomes, patient safety, and programs for the development and effective, safe, and sustained use of medicines are the features of the journal, which

has also been accepted for indexing on PubMed Central. The manuscript management system is completely online and includes a very quick and fair peer-review system, which is all easy to use. Visit http://www.dovepress.com/testimonials.php to read real quotes from published authors.

Submit your manuscript here: http://www.dovepress.com/drug-design-development-and-therapy-journal 